\title{
KETAKSAMAAN INTEGRAL GRONWALL-BELLMAN UNTUK FUNGSI BERPANGKAT
}

(Integral Inequalities of Gronwall-Bellman for Power Function)

\author{
MONALISA ENGELLINE RIJOLY ${ }^{1}$, HENRY JUNUS WATTIMANELA ${ }^{2}$, RUDY WOLTER MATAKUPAN $^{3}$ \\ ${ }^{1}$ Alumni Jurusan Matematika Fakultas MIPA Universitas Pattimura \\ ${ }^{2,3}$ Staf Jurusan Matematika Fakultas MIPA Universitas Pattimura \\ Jl. Ir. M. Putuhena, Kampus Unpatti, Poka-Ambon \\ email: alissangell_ly@yahoo.com; wattimanela@yahoo.com; rwmatakupan@yahoo.com
}

\begin{abstract}
Integral inequality of Gronwall-Bellman is known as an integral inequality which consists of differential and integral forms. Integral inequality of Gronwall-Bellman involving several functions that some definite condition hold and integral values of these functions. In addition, the integral inequality of Gronwall-Bellman shows that if a function is bounded to a certain integral values then that function is also bounded for the other conditions, that is the exponential of integral. Furthermore, by adding some specific conditions the integral inequality of Gronwall-Bellman can be extended to the case of power functions.
\end{abstract}

Keywords: Integral Inequalities Of Gronwall-Bellman, Power Function

\section{PENDAHULUAN}

Dalam perkembangan ilmu Matematika, ketaksamaan memainkan peran yang sangat penting, khususnya dalam bidang analisis. Banyak teori-teori tentang ketaksamaan yang dikembangkan, diantaranya yang sudah dikenal adalah ketaksamaan segitiga, ketaksamaan Cauchy-Schwarz, ketaksamaan Holder dan ketaksamaan Minkowoski.

Teori ketaksamaan lain yang cukup penting adalah ketaksamaan integral. Ketaksamaan ini merupakan salah satu teori yang sangat dibutuhkan dalam studi persamaan diferensial karena dapat digunakan untuk meyelesaikan masalah nilai batas serta dapat menganalisis eksistensi, ketunggalan dan stabilitas dari solusi persamaan diferensial tersebut.

Salah satu ketaksamaan integral yang sangat dikenal adalah ketaksamaan integral Gronwall-Bellman. Ketaksamaan integral Gronwall-Bellman terdiri dari dua bentuk yaitu bentuk diferensial dan bentuk integral. Kedua bentuk ini pertama kali diperkenalkan oleh Thomas Hakon Gronwall pada tahun 1919 dalam tulisannya yang berjudul "Note On The Derivatives With Respects To A Parameter Of The Solutions Of A System Of Differential Equations". Dalam tulisannya Gronwall hanya mampu membuktikan bentuk diferensial sedangkan sedangkan bentuk integralnya dibiarkan tanpa bukti. Kemudian pada tahun 1943 bentuk integral (yang diperkenalkan oleh
Gronwall) berhasil dibuktikan oleh Richard Bellman. Oleh karena keberhasilannya maka ketaksamaan ini dinamakan sebagai ketaksamaan integral GronwallBellman.

Secara umum, konsep ketaksamaan integral Gronwall-Bellman melibatkan beberapa fungsi yang memenuhi syarat tertentu dan nilai integral dari fungsifungsi tersebut. Di sisi lain, ternyata dengan menambahkan beberapa syarat lagi maka ketaksamaan integral Gronwall-Bellman dapat diperluas untuk kasus fungsi berpangkat.

Berdasarkan paparan di atas, maka dalam penelitian ini akan dibahas tentang ketaksamaan integral GronwallBellman untuk fungsi berpangkat.

\section{TINJAUAN PUSTAKA}

Pada tahun 1919, T. H Gronwall menemukan konsep ketaksamaan integral saat sedang mempelajari ketergantungan sistem persamaan diferensial terhadap parameter. Ketaksamaan ini kemudian dikenal sebagai ketaksamaan integral Gronwall. Selanjutnya pada tahun 1943 dalam bukunya yang berjudul "Stability Theory of Differential Equations" R. Bellman menggunakan ketaksamaan Gronwall untuk menyusun sifat-sifat ketaksamaan yang baru, yang dikenal sebagai ketaksamaan integral Gronwall-Bellman. 
Drumi Bainov dan Pavel Simeonov (1991) dalam bukunya yang berjudul "Integral Inequalities and Applications" mengkaji ulang ketaksamaan integral Gronwall-Bellman sehingga lebih sederhana dan mudah dimengerti. Drumi dan Pavel juga membahas aplikasi dari ketaksamaan Gronwall-Bellman dalam persamaan diferensial.

Kemudian H. El-Owady, A. Ragab dan A. Abdeldaim (1999) dalam jurnalnya yang berjudul "On Some New Integral Inequalities of Gronwall-Bellman type" menyusun sifat-sifat ketaksamaan yang baru khususnya untuk fungsi berpangkat.

Dengan menggunakan dua sumber utama di atas dan didukung oleh beberapa literatur yang lain, maka peneliti mencoba menyusun penelitian tentang "Ketaksamaan Integral Gronwall-Bellman Untuk Fungsi Berpangkat' dengan harapan dapat mudah dimengerti.

\section{HASIL DAN PEMBAHASAN}

Pada bagian ini akan dibahas beberapa teorema yang memperlihatkan sifat-sifat dari ketaksamaan integral Gronwall-Bellman dan dilanjutkan dengan membahas perluasan dari ketaksamaan ini untuk fungsi berpangkat.

\section{Ketaksamaan Integral Gronwall-Bellman}

Teorema 1.1 berikut ini merupakan bentuk integral yang diperkenalkan oleh Gronwall dan berhasil dibuktikan oleh Bellman. Teorema ini sangat penting dan juga menjadi dasar dalam pengembangan ketaksamaan integral Gronwall-Bellman.

\section{Teorema 1.1.}

Misalkan $u: \mathbb{R} \rightarrow \mathbb{R}$ dan $b: \mathbb{R} \rightarrow \mathbb{R}$. Misalkan juga $u(t)$ dan $b(t)$ adalah fungsi kontinu yang non negatif untuk $t \geq \alpha$ dimana $\alpha \in \mathbb{R}$. Jika $a \geq 0$ adalah konstanta dan berlaku

$$
u(t) \leq a+\int_{\alpha}^{t} b(s) u(s) d s, \quad t \geq \alpha
$$

maka

$$
u(t) \leq a \exp \left(\int_{\alpha}^{t} b(s) d s\right), \quad t \geq \alpha
$$

\section{Bukti :}

i. Jika $a>0$ maka dari Persamaan (1) dapat diperoleh

$$
\frac{u(t)}{a+\int^{t} b(s) u(s) d s} \leq 1, \quad t \geq \alpha
$$

Misalkan $\tau \geq \alpha$ maka Persamaan (3) dapat ditulis sebagai

$$
\frac{u(\tau)}{a+\int_{\alpha}^{\tau} b(s) u(s) d s} \leq 1
$$

Selanjutnya kalikan $b(\tau)$ terhadap kedua ruas maka diperoleh $\frac{b(\tau) u(\tau)}{a+\int_{\alpha}^{\tau} b(s) u(s) d s} \leq b(\tau)$

$\frac{d}{d \tau} \ln \left(a+\int_{\alpha}^{\tau} b(s) u(s) d s\right) \leq b(\tau)$

Integralkan kedua ruas dari $\alpha$ ke $t$ maka diperoleh

$\int_{\alpha}^{t} \frac{d}{d \tau} \ln \left(a+\int_{\alpha}^{\tau} b(s) u(s) d s\right) d \tau \leq \int_{\alpha}^{t} b(s) d s$

$\left.\ln \left(a+\int_{\alpha}^{\tau} b(s) u(s) d s\right)\right|_{\alpha} ^{t} \leq \int_{\alpha}^{t} b(s) d s$

$\ln \left(a+\int_{\alpha}^{t} b(s) u(s) d s\right)-\ln (a+0) \leq \int_{\alpha}^{t} b(s) d s$

$\ln \left(a+\int_{\alpha}^{t} b(s) u(s) d s\right)-\ln a \leq \int_{\alpha}^{t} b(s) d s$

$\ln \frac{\left(a+\int_{\alpha}^{t} b(s) u(s) d s\right)}{a} \leq \int_{\alpha}^{t} b(s) d s$

$\exp \left[\ln \frac{\left(a+\int_{\alpha}^{t} b(s) u(s) d s\right)}{a}\right] \leq \exp \left(\int_{\alpha}^{t} b(s) d s\right)$

$\frac{\left(a+\int_{\alpha}^{t} b(s) u(s) d s\right)}{a} \leq \exp \left(\int_{\alpha}^{t} b(s) d s\right)$

$a+\int_{\alpha}^{t} b(s) u(s) d s \leq a \exp \left(\int_{\alpha}^{t} b(s) d s\right)$

Berdasarkan Persamaan (1) dan hasil di atas maka diperoleh

$$
u(t) \leq a \exp \left(\int_{\alpha}^{t} b(s) d s\right), \quad t \geq \alpha
$$

ii. Jika $a=0$ maka untuk setiap $\varepsilon>0$ berlaku

$$
u(t) \leq \varepsilon+\int_{\alpha}^{t} b(s) u(s) d s
$$

Dengan menggunakan hasil Bagian i. maka diperoleh

$$
u(t) \leq \varepsilon \exp \left(\int_{\alpha}^{t} b(s) d s\right)
$$

Selanjutnya jika $\varepsilon \rightarrow 0$ maka diperoleh $u(t)=0$.

Selanjutnya dalam pembahasan ini setiap fungsi yang diberikan merupakan fungsi bernilai riil. Sifat berikut ini memperlihatkan akibat yang bisa diperoleh dari Teorema 1.1 di atas jika terdapat dua konstanta dalam ketaksamaan. 
Akibat 1.2.

Misalkan $u(t)$ dan $b(t)$ adalah fungsi non negatif untuk $t \geq \alpha$ dimana $\alpha \in \mathbb{R}$. Jika $a \geq 0$ dan $\gamma$ adalah konstanta,

serta berlaku

$u(t) \leq a e^{-\gamma(t-\alpha)}+\int_{\alpha}^{t} e^{-\gamma(t-s)} b(s) u(s) d s, \quad t \geq \alpha$

maka

$$
\begin{aligned}
u(t) & \leq a \exp \left(-\gamma(t-\alpha)+\int_{\alpha}^{t} b(s) d s\right), \quad t \geq \alpha \\
\ln \left(a+\int_{\alpha}^{t} b(s) u(s) d s\right) & -\ln \left(a+\int_{\alpha}^{\alpha} b(s) u(s) d s\right) \leq \int_{\alpha}^{t} b(s) d s
\end{aligned}
$$

\section{Bukti :}

Dari Persamaan (4) dapat diperoleh

$$
\begin{gathered}
u(t) \leq a e^{-\gamma t+\gamma \alpha}+\int_{\alpha}^{t} e^{-\gamma t+\gamma s} b(s) u(s) d s \\
u(t) \leq e^{-\gamma t}\left(a e^{\gamma \alpha}+\int_{\alpha}^{t} e^{\gamma s} b(s) u(s) d s\right) \\
e^{\gamma t} u(t) \leq a e^{\gamma \alpha}+\int_{\alpha}^{t} e^{\gamma s} b(s) u(s) d s
\end{gathered}
$$

Misalkan $w(t)=e^{\gamma t} u(t)$ maka diperoleh

$$
w(t) \leq a e^{\gamma \alpha}+\int_{\alpha}^{t} b(s) w(s) d s
$$

Berdasarkan Teorema 1.1. maka diperoleh

$$
w(t) \leq a e^{\gamma \alpha} \exp \left(\int_{\alpha}^{t} b(s) d s\right)
$$

Karena $w(t)=e^{\gamma t} u(t)$ maka

$$
u(t) \leq a e^{-\gamma t} e^{\gamma \alpha} \exp \left(\int_{\alpha}^{t} b(s) d s\right)
$$

sehingga terbukti $u(t) \leq a e^{-\gamma(t-\alpha)} \exp \left(\int_{\alpha}^{t} b(s) d s\right)$ atau

$$
u(t) \leq a \exp \left((-\gamma(t-\alpha))+\int_{\alpha}^{t} b(s) d s\right)
$$

Teorema 1.3. berikut ini merupakan bentuk diferensial yang diperkenalkan dan dibuktikan oleh Gronwall. Sama seperti Teorema 1.1. di atas, teorema ini sangat penting dalam pengembangan ketaksamaan integral Gronwall-Bellman.

Teorema 1.3.

Misalkan $b(t)$ dan $f(t)$ adalah fungsi kontinu untuk $t \geq \alpha$ dimana $\alpha \in \mathbb{R}$ dan $v(t)$ adalah fungsi yang terdiferensiasi untuk $t \geq \alpha$.

Jika berlaku $v^{\prime}(t) \leq b(t) v(t)+f(t), t \geq \alpha$

dan

$v(\alpha) \leq a$, dimana $a$ konstanta

$v(t) \leq a \exp \left(\int_{a}^{t} b(s) d s\right)$

$$
+\int_{\alpha}^{t} f(s) \exp \left(\int_{s}^{t} b(\tau) d \tau\right) d s
$$

\section{Bukti :}

Misalkan $s \geq \alpha$ maka dari Persamaan (6) dapat diperoleh $v^{\prime}(s)-b(s) v(s) \leq f(s)$

$$
\begin{array}{r}
{\left[v^{\prime}(s)-b(s) v(s)\right] \exp \left(\int_{s}^{t} b(\tau) d \tau\right)} \\
\leq f(s) \exp \left(\int_{s}^{t} b(\tau) d \tau\right) \\
v^{\prime}(s) \exp \left(\int_{s}^{t} b(\tau) d \tau\right)-b(s) v(s) \exp \left(\int_{s}^{t} b(\tau) d \tau\right) \\
\leq f(s) \exp \left(\int_{s}^{t} b(\tau) d \tau\right) \\
\frac{d}{d s}\left[v(s) \exp \left(\int_{s}^{t} b(\tau) d \tau\right)\right] \leq f(s) \exp \left(\int_{s}^{t} b(\tau) d \tau\right)
\end{array}
$$

Integralkan kedua ruas dari $\alpha$ ke $t$ maka diperoleh

$$
\begin{aligned}
& \int_{\alpha}^{t} \frac{d}{d s}\left[v(s) \exp \left(\int_{s}^{t} b(\tau) d \tau\right)\right] d s \\
& \leq \int_{\alpha}^{t} f(s) \exp \left(\int_{s}^{t} b(\tau) d \tau\right) d s
\end{aligned}
$$

$\left.v(s) \exp \left(\int_{s}^{t} b(\tau) d \tau\right)\right|_{\alpha} ^{t}$

$$
\begin{aligned}
& \leq \int_{\alpha}^{t} f(s) \exp \left(\int_{s}^{t} b(\tau) d \tau\right) d s \\
& v(t) \exp \left(\int_{t}^{t} b(\tau) d \tau\right)-v(\alpha) \exp \left(\int_{\alpha}^{t} b(\tau) d \tau\right) \\
& \leq \int_{\alpha}^{t} f(s) \exp \left(\int_{s}^{t} b(\tau) d \tau\right) d s \\
& v(t) \exp (0)-v(\alpha) \exp \left(\int_{\alpha}^{t} b(\tau) d \tau\right) \\
& \leq \int_{\alpha}^{t} f(s) \exp \left(\int_{s}^{t} b(\tau) d \tau\right) d s \\
& v(t) \leq v(\alpha) \exp \left(\int_{\alpha}^{t} b(\tau) d \tau\right) \\
& +\int_{\alpha}^{t} f(s) \exp \left(\int_{s}^{t} b(\tau) d \tau\right) d s
\end{aligned}
$$


Karena $v(\alpha) \leq a$ maka terbukti

$v(t) \leq a \exp \left(\int_{\alpha}^{t} b(s) d s\right)+\int_{\alpha}^{t} f(s) \exp \left(\int_{s}^{t} b(\tau) d \tau\right) d s$

\section{Teorema 1.4.}

Misalkan $b(t)$ dan $u(t)$ adalah fungsi non negatif yang kontinu pada $J=[\alpha, \beta]$ dimana $\alpha, \beta \in \mathbb{R}$. Jika $a \geq 0$ dan berlaku

$u(t) \leq a+\int_{t}^{t} b(s) u(s)|d s|, \quad t, t_{0} \in J$

maka

$$
u(t) \leq a \exp \left(\int_{t_{0}}^{t} b(s)|d s|\right), \quad t, t_{0} \in J
$$

\section{Bukti :}

Nilai mutlak pada Persamaan (9) akan menjamin $\int^{t} b(s) u(s)|d s|$ bernilai non negatif, sehingga dengan

Teorema 1.1. maka Teorema 1.4. terbukti.

\section{Teorema 1.5.}

Misalkan $a(t), b(t)$ dan $u(t)$ adalah fungsi kontinu pada $J=[\alpha, \beta]$ dimana $\alpha, \beta \in \mathbb{R} \quad$ dan $b(t)$ adalah fungsi non negatif pada $J$.

Jika berlaku

$$
u(t) \leq a(t)+\int_{\alpha}^{t} b(s) u(s) d s, \quad t \in J
$$

maka

$$
\begin{aligned}
u(t) \leq & a(t) \\
& +\int_{\alpha}^{t} a(s) b(s) \exp \left(\int_{s}^{t} b(\tau) d \tau\right) d s, \quad t \in J
\end{aligned}
$$

\section{Bukti :}

Misalkan $v(t)=\int^{t} b(s) u(s) d s$, maka

$$
u(t) \leq a(t)+v(t)
$$

dan jelas bahwa $v(\alpha)=0$. Selanjutnya

$$
\begin{aligned}
v^{\prime}(t) & =b(t) u(t) \\
& \leq b(t)[a(t)+v(t)] \\
& =b(t) v(t)+a(t) b(t), \quad t \in J
\end{aligned}
$$

Misalkan bahwa $f(t)=a(t) b(t)$ maka

$$
v^{\prime}(t) \leq b(t) v(t)+f(t)
$$

Dengan menggunakan Teorema 1.3. diperoleh

$$
\begin{aligned}
v(t) \leq v(\alpha) & \exp \left(\int_{\alpha}^{t} b(\tau) d \tau\right) \\
& +\int_{\alpha}^{t} f(s) \exp \left(\int_{s}^{t} b(\tau) d \tau\right) d s
\end{aligned}
$$

$$
\begin{aligned}
& v(t) \leq(0) \exp \left(\int_{\alpha}^{t} b(\tau) d \tau\right) \\
& +\int_{\alpha}^{t} a(s) b(s) \exp \left(\int_{s}^{t} b(\tau) d \tau\right) d s \\
& v(t) \leq \int_{\alpha}^{t} a(s) b(s) \exp \left(\int_{s}^{t} b(\tau) d \tau\right) d s
\end{aligned}
$$

Karena $u(t) \leq a(t)+v(t)$ maka terbukti

$u(t) \leq a(t)+\int_{\alpha}^{t} a(s) b(s) \exp \left(\int_{s}^{t} b(\tau) d \tau\right) d s$

Berdasarkan Teorema 1.5 dapat diperoleh beberapa akibat yang dperlihatkan dalam sifat-sifat berikut ini.

\section{Akibat 1.6.}

Misalkan jika dalam Teorema 1.5. fungsi $a(t)$ juga merupakan fungsi tidak turun dalam $J$, maka berlaku

$$
u(t) \leq a(t) \exp \left(\int_{\alpha}^{t} b(s) d s\right), \quad t \in J
$$

\section{Bukti :}

Karena $a(t)$ juga merupakan fungsi tidak turun dalam $J$ maka Persamaan (12) dapat ditulis sebagai

$$
\begin{aligned}
u(t) & \leq a(t)+a(t) \int_{\alpha}^{t} b(s) \exp \left(\int_{s}^{t} b(\tau) d \tau\right) d s \\
& =a(t)\left(1+\int_{\alpha}^{t} b(s) \exp \left(\int_{s}^{t} b(\tau) d \tau\right) d s\right) \\
& =a(t)\left(1-\int_{\alpha}^{t} \frac{d}{d s}\left[\exp \left(\int_{s}^{t} b(\tau) d \tau\right)\right] d s\right) \\
& \left.=\left.a(t)\left(1-\left(\exp \left(\int_{s}^{t} b(\tau) d \tau\right)\right) \mid t\right)\right|_{\alpha}\right) \\
& =a(t)\left(1-\left(\exp \left(\int_{t}^{t} b(\tau) d \tau\right)-\exp \left(\int_{\alpha}^{t} b(\tau) d \tau\right)\right)\right. \\
& =a(t)\left(1-\left(\exp (0)-\exp \left(\int_{\alpha}^{t} b(\tau) d \tau\right)\right)\right) \\
& =a(t) \exp \left(\int_{\alpha}^{t} b(\tau) d \tau\right) \\
& a(t)\left(1+\exp \left(\int_{\alpha}^{t} b(\tau) d \tau\right)\right)
\end{aligned}
$$

atau dapat ditulis

$$
u(t) \leq a(t) \exp \left(\int_{\alpha}^{t} b(s) d s\right), t \in J
$$

\section{Akibat 1.7.}

Misalkan $b(t)$ dan $u(t)$ adalah fungsi kontinu pada $J=[\alpha, \beta]$ dimana $\alpha, \beta \in \mathbb{R}$. 
Jika $b(t)$ adalah fungsi non negatif pada $J$ dan $a$ adalah konstanta serta berlaku

$$
u(t) \leq a+\int_{\alpha}^{t} b(s) u(s) d s \quad, \quad t \in J
$$

maka

$$
u(t) \leq a \exp \left(\int_{\alpha}^{t} b(s) d s\right), \quad t \in J
$$

\section{Bukti :}

Jelas dengan menggunakan Akibat 1.6.

\section{Akibat 1.8.}

Misalkan $u(t)$ adalah fungsi kontinu pada $J=[\alpha, \beta]$ dimana $\alpha, \beta \in \mathbb{R}$. Jika $a$ dan $b>0$ adalah konstanta serta berlaku

$$
u(t) \leq a+\int_{\alpha}^{t} b u(s) d s, \quad t \in J
$$

maka

$$
u(t) \leq a e^{b t-\alpha}, \quad t \in J
$$

Bukti :

Berdasarkan Akibat 1.7 diperoleh

$$
\begin{aligned}
u(t) & \leq a \exp \left(\int_{\alpha}^{t} b d s\right) \\
& =a \exp (b(t-\alpha)) \\
& =a e^{b(t-\alpha)}
\end{aligned}
$$

\section{Akibat 1.9.}

Misalkan $a(t), b(t), c(t)$ dan $u(t)$ adalah fungsi kontinu pada $J=[\alpha, \beta]$ dimana $\alpha, \beta \in \mathbb{R}$. Jika $b(t)$ dan $c(t)$ adalah fungsi non negatif pada $J$ dan berlaku

$$
u(t) \leq a(t)+\int_{\alpha}^{t}(b(s) u(s)+c(s)) d s
$$

maka

$u(t) \leq\left[\sup _{s \in[\alpha, t]} a(s)+\int_{\alpha}^{t} c(s) d s\right] \exp \left(\int_{\alpha}^{t} b(s) d s\right), \quad t \in J$

Bukti :

Dari Persamaan (13) dapat diperoleh

$u(t) \leq a(t)+\int_{\alpha}^{t} b(s) u(s) d s+\int_{\alpha}^{t} c(s) d s$

$u(t) \leq \sup _{s \in[\alpha, t]} a(s)+\int_{\alpha}^{t} c(s) d s+\int_{\alpha}^{t} b(s) u(s) d s$

Misalkan $A(t)=\sup _{s \in[\alpha, t]} a(s)+\int_{\alpha}^{t} c(s) d s$ maka hasil di atas dapat ditulis menjadi $u(t) \leq A(t)+\int^{t} b(s) u(s) d s$
Karena $A(t)=\sup _{s \in[\alpha, t]} a(s)+\int^{t} c(s) d s$ adalah fungsi tidak turun pada $J$ maka berdasarkan Akibat 1.6. diperoleh

$u(t) \leq A(t) \exp \left(\int_{\alpha}^{t} b(s) d s\right), \quad t \in J$

atau terbukti bahwa

$$
u(t) \leq\left[\sup _{s \in[\alpha, t]} a(s)+\int_{\alpha}^{t} c(s) d s\right] \exp \left(\int_{\alpha}^{t} b(s) d s\right)
$$

untuk $t \in J$ 口

Sifat berikut ini merupakan penyempurnaan dari Akibat 1.9.

\section{Teorema 1.10.}

Misalkan $a(t), b(t), c(t)$ dan $u(t)$ adalah fungsi kontinu pada $J=[\alpha, \beta]$ dimana $\alpha, \beta \in \mathbb{R}$. Jika $b(t)$ adalah fungsi non negatif pada $J$ dan berlaku

$u(t) \leq a(t)+\int_{\alpha}^{t}(b(s) u(s)+c(s)) d s, \quad t \in J$

maka

$$
\begin{aligned}
a(t) \leq a(t)+\int_{\alpha}^{t}\{[a(s) b(s)+c(s)] \\
\left.\quad \exp \left(\int_{s}^{t} b(\tau) d \tau\right)\right\} d s, t \in J
\end{aligned}
$$

Bukti :

Misalkan $v(t)=\int_{\alpha}^{t}[b(s) u(s)+c(s)] d s$ maka $u(t) \leq a(t)+v(t)$ dan jelas bahwa $v(\alpha)=0$. Selanjutnya

$$
\begin{aligned}
v^{\prime}(t) & =b(t) u(t)+c(t) \\
& \leq b(t)[a(t)+v(t)]+c(t) \\
& =b(t) v(t)+a(t) b(t)+c(t)
\end{aligned}
$$

Misalkan $f(t)=a(t) b(t)+c(t)$ maka $v^{\prime}(t)=b(t) v(t)+f(t) \quad$ sehingga berdasarkan Teorema 1.3. diperoleh

$$
\begin{aligned}
& v(t) \leq v(\alpha) \exp \left(\int_{\alpha}^{t} b(\tau) d \tau\right) \\
& +\int_{\alpha}^{t} f(s) \exp \left(\int_{s}^{t} b(\tau) d \tau\right) d s \\
& v(t) \leq(0) \exp \left(\int_{a}^{t} b(\tau) d \tau\right) \\
& +\int_{\alpha}^{t}[a(s) b(s)+c(s)] \exp \left(\int_{s}^{t} b(\tau) d \tau\right) d s
\end{aligned}
$$


$v(t) \leq \int_{\alpha}^{t}[a(s) b(s)+c(s)] \exp \left(\int_{s}^{t} b(\tau) d \tau\right) d s$

Karena $u(t) \leq a(t)+v(t)$

maka didapat

$u(t) \leq a(t)$

$$
+\int_{\alpha}^{t}[a(s) b(s)+c(s)] \exp \left(\int_{s}^{t} b(\tau) d \tau\right) d s
$$

Akibat 1.11.

Jika pada Teorema 1.10 fungsi $a(t)$ adalah fungsi tidak turun maka berlaku

$$
\begin{aligned}
u(t) \leq a(t) & \exp \left(\int_{\alpha}^{t} b(\tau) d \tau\right) \\
& +\int_{\alpha}^{t} c(s) \exp \left(\int_{s}^{t} b(\tau) d \tau\right) d s
\end{aligned}
$$

Bukti :

$$
\begin{aligned}
u(t) \leq a(t)+ & \int_{\alpha}^{t} a(s) b(s) \exp \left(\int_{s}^{t} b(\tau) d \tau\right) d s \\
& +\int_{\alpha}^{t} c(s) \exp \left(\int_{s}^{t} b(\tau) d \tau\right) d s \\
u(t) \leq a(t)+ & a(t) \int_{\alpha}^{t} b(s) \exp \left(\int_{s}^{t} b(\tau) d \tau\right) d s \\
& +\int_{\alpha}^{t} c(s) \exp \left(\int_{s}^{t} b(\tau) d \tau\right) d s
\end{aligned}
$$

Dengan menggunakan hasil pada Akibat 1.6 maka diperoleh

$$
\begin{aligned}
u(t) \leq a(t) & \exp \left(\int_{\alpha}^{t} b(\tau) d \tau\right) \\
& +\int_{\alpha}^{t} c(s) \exp \left(\int_{s}^{t} b(\tau) d \tau\right) d s \quad \square
\end{aligned}
$$

\section{Akibat 1.12.}

Jika pada Teorema 1.10 , fungsi $a(t)$ diganti dengan konstanta $d$ maka berlaku

$$
\begin{aligned}
u(t) \leq d & \exp \left(\int_{\alpha}^{t} b(\tau) d \tau\right) \\
& +\int_{\alpha}^{t} c(s) \exp \left(\int_{s}^{t} b(\tau) d \tau\right) d s, \quad t \in J
\end{aligned}
$$

Bukti :

Jelas dengan menggunakan Akibat 1.11.

\section{Akibat 1.13.}

Misalkan $u(t)$ adalah fungsi kontinu untuk $t \geq \alpha$ dimana $\alpha \in \mathbb{R}$. Jika $b>0, a, c$ dan $\gamma \neq b$ adalah konstanta dan berlaku $u(t) \leq a e^{-\gamma(t-\alpha)}+\int_{\alpha}^{t} e^{-\gamma(t-s)}[b u(s)+c] d s, \quad t \geq \alpha$

maka

$u(t) \leq a e^{(b-\gamma)(t-\alpha)}+\frac{c}{\gamma-b}\left(1-e^{(b-\gamma)(t-\alpha)}\right), \quad t \geq \alpha$

\section{Bukti :}

$$
\begin{aligned}
& u(t) \leq a e^{-\gamma(t-\alpha)} \exp \left(\int_{\alpha}^{t} b d \tau\right)+\int_{\alpha}^{t} e^{-\gamma(t-s)} c \exp \left(\int_{s}^{t} b d \tau\right) d s \\
& =a e^{-\gamma(t-\alpha)} \exp (b(t-\alpha)) \\
& +\int_{\alpha}^{t} e^{-\gamma(t-s)} c \exp (b(t-s)) d s \\
& =a e^{-\gamma(t-\alpha)} e^{b(t-\alpha)}+\int_{\alpha}^{t} e^{-\gamma(t-s)} c e^{b(t-s)} d s \\
& =a e^{(b-\gamma)(t-\alpha)}+\int_{\alpha}^{t} c e^{(b-\gamma)(t-s)} d s \\
& =a e^{(b-\gamma)(t-\alpha)}+\int_{\alpha}^{t} c e^{(b-\gamma)(t-s)} d s \\
& =a e^{(b-\gamma)(t-\alpha)}+\left.\left(\frac{c}{(\gamma-b)} e^{(b-\gamma)(t-s)}\right)\right|_{\alpha} ^{t} \\
& =a e^{(b-\gamma)(t-\alpha)}+\left(\left(\frac{c}{(\gamma-b)} e^{(b-\gamma)(t-\alpha)}\right)\right. \\
& \left.-\left(\frac{c}{(\gamma-b)} e^{(b-\gamma)(t-\alpha)}\right)\right) \\
& =a e^{(b-\gamma)(t-\alpha)}+\frac{c}{(\gamma-b)}\left(1-e^{(b-\gamma)(t-\alpha)}\right)
\end{aligned}
$$

Sehingga

$u(t) \leq a e^{(b-\gamma)(t-\alpha)}+\frac{c}{\gamma-b}\left(1-e^{(b-\gamma)(t-\alpha)}\right), t \geq \alpha$

\section{Akibat 1.14.}

Misalkan $u(t)$ adalah fungsi kontinu pada $J=[\alpha, \beta]$ dimana $\alpha, \beta \in \mathbb{R}$. Jika $b>0, a$, dan $c$ adalah konstanta dan berlaku

$u(t) \leq a+\int_{\alpha}^{t}(b u(s)+c) d s, \quad t \in J$

maka

$u(t) \leq a e^{b(t-\alpha)}+\frac{c}{b}\left(e^{b(t-\alpha)}-1\right), \quad t \in J$

\section{Bukti :}

Dengan menggunakan Akibat 1.13. dan diambil $\gamma=0$ maka diperoleh 


$$
\begin{aligned}
u(t) & \leq a e^{(b-\gamma)(t-\alpha)}+\frac{c}{\gamma-b}\left(1-e^{(b-\gamma)(t-\alpha)}\right) \\
& =a e^{(b-0)(t-\alpha)}+\frac{c}{0-b}\left(1-e^{(b-0)(t-\alpha)}\right) \\
& =a e^{b(t-\alpha)}-\frac{c}{b}\left(1-e^{b(t-\alpha)}\right) \\
& =a e^{b(t-\alpha)}+\frac{c}{b}\left(e^{b(t-\alpha)}-1\right)
\end{aligned}
$$

sehingga $u(t) \leq a e^{b(t-\alpha)}+\frac{c}{b}\left(e^{b(t-\alpha)}-1\right), t \in J$

\section{Teorema 1.15.}

Misalkan $u(t)$ dan $b(t)$ fungsi kontinu pada $J=[\alpha, \beta]$ dimana $\alpha, \beta \in \mathbb{R}$ sedangkan $a(t)$ dan $q(t)$ fungsi yang terintegral Rieman pada $J$. Misalkan juga $b(t)$ dan $q(t)$ adalah fungsi non negatif pada $J$.

Jika $u(t) \leq a(t)+q(t) \int_{\alpha}^{t} b(s) u(s) d s, \quad t \in J$ maka

$$
\begin{aligned}
u(t) \leq a(t)+ & q(t)\left[\int_{\alpha}^{t} a(s) b(s)\right. \\
& \left.\quad \exp \left(\int_{s}^{t} b(\tau) q(\tau) d \tau\right) d s\right], t \in J
\end{aligned}
$$

\section{Bukti :}

Misalkan $v(t)=\int_{\alpha}^{t} b(s) u(s) d s$ maka

$$
u(t) \leq a(t)+q(t) v(t)
$$

dan jelas bahwa $v(\alpha)=0$. Selanjutnya

$$
\begin{aligned}
v^{\prime}(t) & =b(t) u(t) \\
& \leq b(t)[a(t)+q(t) v(t)] \\
& =b(t) q(t) v(t)+a(t) b(t)
\end{aligned}
$$

Misalkan $h(t)=b(t) q(t)$ dan $f(t)=b(t) a(t)$ maka $v^{\prime}(t) \leq h(t) v(t)+f(t) \quad$ sehingga berdasarkan Teorema 1.3. diperoleh $v(t) \leq \int_{\alpha}^{t} a(s) b(s) \exp \left(\int_{s}^{t} b(\tau) q(\tau) d \tau\right) d s, \quad t \in J$. Karena $u(t) \leq a(t)+q(t) v(t)$ maka diperoleh

$$
\begin{aligned}
u(t) \leq & a(t)+q(t) \\
& \left(\int_{\alpha}^{t} a(s) b(s) \exp \left(\int_{s}^{t} b(\tau) q(\tau) d \tau\right) d s\right)
\end{aligned}
$$

dimana $t \in J \square$

\section{Ketaksamaan Integral Gronwall-Bellman Untuk Fungsi Berpangkat}

Dalam Bagian 1. sebelumnya telah diperlihatkan sifat-sifat dasar dari ketaksamaan integral GronwallBellman. Dalam bagian ini akan diperlihatkan beberapa sifat-sifat ketaksamaan integral Gronwall-Bellman yang baru khususnya untuk fungsi berpangkat.

\section{Teorema 2.1}

Misalkan $u(t)$ adalah fungsi yang positif dan kontinu pada $J=[0, \infty)$. Misalkan juga $b(t)$ adalah fungsi non negatif yang kontinu pada $J$. Jika $p \geq 2$ dan $a$ adalah konstanta positif serta berlaku

$$
u^{p}(t) \leq a+\int_{0}^{t} b(s) u(s) d s, \quad t \in J
$$

maka

$u(t) \leq\left[a^{\frac{q}{p}}+\frac{q}{p} \int_{0}^{t} b(s) d s\right]^{\frac{1}{q}}, t \in J$

dimana $p-q=1$.

\section{Bukti :}

Berdasarkan (1), diferensialkan $u^{p}(t)$ terhadap $t$

$\frac{d}{d t}\left[u^{p}(t)\right] \leq \frac{d}{d t}\left[a+\int_{0}^{t} b(s) u(s) d s\right]$

$p u^{p-1}(t) \leq b(t) u(t)$ maka $p u^{(p-2)}(t) \leq b(t)$

Integralkan kedua ruas dari 0 ke $t$

$\int_{0}^{t} p u^{(p-2)}(t) d t \leq \int_{0}^{t} b(s) d s$

$\left.\frac{p}{(p-2)+1} u^{(p-2)+1}(t)\right|_{0} ^{t} \leq \int_{0}^{t} b(s) d s$

$\left.\frac{p}{p-1} u^{(p-1)}(t)\right|_{0} ^{t} \leq \int_{0}^{t} b(s) d s$

karena $p-q=1$ maka $q=p-1$ sehingga dapat ditulis $\left.\frac{p}{q} u^{q}(t)\right|_{0} ^{t} \leq \int_{0}^{t} b(s) d s$

$\frac{p}{q} u^{q}(t)-\frac{p}{q} u^{q}(0) \leq \int_{0}^{t} b(s) d s$

$\frac{p}{q} u^{q}(t) \leq \frac{p}{q} u^{q}(0)+\int_{0}^{t} b(s) d s$

Berdasarkan (1), $u^{p}(0) \leq a$ maka $u^{q}(0) \leq a^{\frac{q}{p}}$ sehingga

$$
\frac{p}{q} u^{q}(t) \leq \frac{p}{q} a^{\frac{q}{p}}+\int_{0}^{t} b(s) d s
$$

Kedua ruas dikalikan dengan $\frac{q}{p}$, sehingga diperoleh

$$
u^{q}(t) \leq a^{\frac{q}{p}}+\frac{q}{p} \int_{0}^{t} b(s) d s
$$


kemudian kedua ruas dipangkatkan $\frac{1}{q}$, sehingga

diperoleh $u(t) \leq\left[a^{\frac{q}{p}}+\frac{q}{p} \int_{0}^{t} b(s) d s\right]^{\frac{1}{q}}$

\section{Teorema 2.2}

Misalkan $u(t)$ dan $b(t)$ adalah fungsi non negatif yang kontinu pada $J=[0, \infty)$. Misalkan juga $a(t)$ adalah fungsi positif, monoton, tidak turun dan kontinu pada $J$. Jika $p \geq 2$ adalah konstanta dan berlaku

$u^{p}(t) \leq a^{p}(t)+\int_{0}^{t} b(s) u(s) d s, \quad t \in J$

maka

$u(t) \leq a(t)\left[1+\frac{q}{p} \int_{0}^{t} b(s) a^{-q}(s) d s\right]^{\frac{1}{q}}, t \in J$

dimana $p-q=1$.

\section{Bukti :}

Karena $a(t)$ adalah fungsi positif, monoton, dan tidak turun maka Persamaan (3) dapat ditulis sebagai

$$
\begin{aligned}
& \frac{u^{p}(t)}{a^{p}(t)} \leq 1+\int_{0}^{t} b(s)\left[\frac{u(s)}{a^{p}(t)}\right] d s \\
& {\left[\frac{u(t)}{a(t)}\right]^{p} \leq 1+\int_{0}^{t} b(s)\left[\frac{u(s)}{a^{p}(t)}\right] d s}
\end{aligned}
$$

Karena $p-q=1$ maka $p=1+q$ sehingga dapat ditulis

$$
\begin{aligned}
& {\left[\frac{u(t)}{a(t)}\right]^{p} \leq 1+\int_{0}^{t} b(s)\left[\frac{u(s)}{a^{(1+q)}(s)}\right] d s} \\
& {\left[\frac{u(t)}{a(t)}\right]^{p} \leq 1+\int_{0}^{t} b(s)\left[\frac{u(s)}{a(s) \cdot a^{q}(s)}\right] d s} \\
& {\left[\frac{u(t)}{a(t)}\right]^{p} \leq 1+\int_{0}^{t} b(s) a^{-q}(s)\left[\frac{u(s)}{a(s)}\right] d s}
\end{aligned}
$$

Misalkan

$$
m(t)=\frac{u(t)}{a(t)}
$$

maka

$$
m^{p}(t) \leq 1+\int_{0}^{t} b(s) a^{-q}(s) m(s) d s \quad, \quad t \in J
$$

Berdasarkan Teorema 2.1 maka dapat diperoleh

$$
\begin{aligned}
& m(t) \leq\left[1+\frac{q}{p} \int_{0}^{t} b(s) a^{-q}(s) d s\right]^{\frac{1}{q}}, t \in J \\
& \frac{u(t)}{a(t)} \leq\left[1+\frac{q}{p} \int_{0}^{t} b(s) a^{-q}(s) d s\right]^{\frac{1}{q}}, t \in J
\end{aligned}
$$

$$
u(t) \leq a(t)\left[1+\frac{q}{p} \int_{0}^{t} b(s) a^{-q}(s) d s\right]^{\frac{1}{q}}, t \in J
$$

\section{Teorema 2.3}

Misalkan $b(t)$ dan $f(t)$ adalah fungsi non negatif yang kontinu pada $J=[0, \infty)$. Jika $v(t)$ adalah fungsi non negatif yang terdiferensiasi dan memenuhi

$v^{\prime}(t) \leq b(t) v(t)+f(t) v^{p}(t), t \in J$

maka berlaku

$$
\begin{aligned}
v(t) \leq & \exp \left(\int_{0}^{t} b(s) d s\right) \\
& {\left[v^{q}(0)+q \int_{0}^{t} f(s) \exp \left(-q \int_{0}^{s} b(\tau) d \tau\right) d s\right]^{\frac{1}{q}} }
\end{aligned}
$$

\section{Bukti :}

Misalkan $z(t)=\frac{v^{q}(t)}{q}$, maka

$$
\begin{aligned}
z^{\prime}(t) & =\left(v^{q-1}(t)\right) v^{\prime}(t) \\
& \leq v^{q-1}(t)\left(b(t) v(t)+f(t) v^{p}(t)\right) \\
& =v^{q}(t) b(t)+f(t) v^{q-1+p}(t) \\
& =q \frac{v^{q}(t)}{q} b(t)+f(t) v^{0}(t)
\end{aligned}
$$

atau $z^{\prime}(t) \leq q b(t) z(t)+f(t)$.

Berdasarkan Teorema 1.3 maka diperoleh

$$
\begin{aligned}
& z(t) \leq z(0) \exp \left(\int_{0}^{t} q b(s) d s\right) \\
& +\int_{0}^{t} f(s) \exp \left(\int_{s}^{t} q b(\tau) d \tau\right) d s \\
& \text { Karena } z(t)=\frac{v^{q}(t)}{q} \text { maka } \\
& \frac{v^{q}(t)}{q} \leq \frac{v^{q}(0)}{q} \exp \left(\int_{0}^{t} q b(s) d s\right) \\
& +\int_{0}^{t} f(s) \exp \left(\int_{s}^{t} q b(\tau) d \tau\right) d s \\
& v^{q}(t) \leq v^{q}(0) \exp \left(\int_{0}^{t} q b(s) d s\right) \\
& +q \int_{0}^{t} f(s) \exp \left(\int_{s}^{t} q b(\tau) d \tau\right) d s
\end{aligned}
$$




$$
\begin{aligned}
& =v^{q}(0) \exp \left(\int_{0}^{t} q b(s) d s\right) \\
& +q \int_{0}^{t} f(s) \exp \left(\int_{0}^{t} q b(\tau) d \tau+\int_{s}^{0} q b(\tau) d \tau\right) d s \\
& =v^{q}(0) \exp \left(\int_{0}^{t} q b(s) d s\right)+\exp \left(\int_{0}^{t} q b(\tau) d \tau\right) \\
& \left.q \int_{0}^{t} f(s) \exp \left(-\int_{0}^{s} q b(\tau) d \tau\right) d s\right] \\
& =v^{q}(0) \exp \left(\int_{0}^{t} q b(s) d s\right)+\exp \left(\int_{0}^{t} q b(s) d s\right) \\
& \left.q \int_{0}^{t} f(s) \exp \left(-\int_{0}^{s} q b(\tau) d \tau\right) d s\right] \\
& =\exp \left(\int_{0}^{t} q b(s) d s\right) \\
& {\left[v^{q}(0)+q \int_{0}^{t} f(s) \exp \left(-\int_{0}^{s} q b(\tau) d \tau\right) d s\right]}
\end{aligned}
$$

sehingga diperoleh

$$
\begin{aligned}
v^{q}(t) \leq & \exp \left(q \int_{0}^{t} b(s) d s\right) \\
& {\left[v^{q}(0)+q \int_{0}^{t} f(s) \exp \left(-q \int_{0}^{s} b(\tau) d \tau\right) d s\right] }
\end{aligned}
$$

Pangkatkan kedua ruas persamaan di atas dengan $\frac{1}{q}$ maka diperoleh

$$
\begin{aligned}
v(t) \leq & {\left[\exp \left(q \int_{0}^{t} b(s) d s\right)\right]^{\frac{1}{q}} } \\
& {\left[v^{q}(0)+q \int_{0}^{t} f(s) \exp \left(-q \int_{0}^{s} b(\tau) d \tau\right) d s\right]^{\frac{1}{q}} } \\
& {\left.\left[v^{q}(0)+q \int_{0}^{t} f(s) d s\right) \exp \left(-q \int_{0}^{s} b(\tau) d \tau\right) d s\right]^{\frac{1}{q}} }
\end{aligned}
$$

atau dapat ditulis

$$
\begin{aligned}
v(t) \leq & \exp \left(\int_{0}^{t} b(s) d s\right) \\
& {\left[v^{q}(0)+q \int_{0}^{t} f(s) \exp \left(-q \int_{0}^{s} b(\tau) d \tau\right) d s\right]^{\frac{1}{q}} }
\end{aligned}
$$

\section{Teorema 2.4}

Misalkan $u(t), b(t)$ dan $c(t)$ adalah fungsi non negatif yang kontinu pada $J=[0, \infty)$. Jika $a$ adalah konstanta non negatif dan untuk $0<p<1$ berlaku $u(t) \leq a+\int_{0}^{t} b(s)\left(u^{p}(s)+\int_{0}^{s} c(\tau) u(\tau) d \tau\right) d s$

dimana $t \in J$, maka

$u(t) \leq a+\int_{0}^{t} b(s) k(s) \exp \left(\int_{0}^{s} c(\tau) d \tau\right) d s$

dimana

$k(t)=\left(a^{p q}+p q \int_{0}^{t} b(s) \exp \left(-q \int_{0}^{s} c(\tau) d \tau\right) d s\right)^{\frac{1}{q}}$

dan

$p+q=1$

Bukti :

Diferensiasikan $u(t)$ terhadap $t$ pada Persamaan (9) maka diperoleh

$u^{\prime}(t) \leq b(t)\left(u^{p}(t)+\int_{0}^{t} c(s) u(s) d s\right), t \in J$

Misalkan $\quad v(t)=u^{p}(t)+\int_{0}^{t} c(s) u(s) d s, t \in J \quad$ maka

Persamaan (12) menjadi

$u^{\prime}(t) \leq b(t) v(t)$

Selanjutnya jelas bahwa

$v(0)=u^{p}(0)$

$$
\leq a^{p}
$$

Diferensiasikan $v(t)$ terhadap $t$ maka diperoleh

$v^{\prime}(t)=p u^{p-1}(t) u^{\prime}(t)+c(t) u(t) a$

$$
\leq p u^{p-1}(t)[b(t) v(t)]+c(t) u(t)
$$

Karena $u(t) \leq v(t)$

$$
\begin{aligned}
& \begin{aligned}
v^{\prime}(t) & \leq p v^{p-1}(t)[b(t) v(t)]+c(t) v(t) \\
& =p v^{p}(t) b(t)+c(t) v(t)
\end{aligned} \\
& \text { atau } \\
& v^{\prime}(t) \leq c(t) v(t)+p b(t) v^{p}(t)
\end{aligned}
$$

Dari Persamaan (14) dan berdasarkan Teorema 2.3 maka untuk $t \in J$ diperoleh

$$
\begin{aligned}
v(t) \leq & \exp \left(\int_{0}^{t} c(s) d s\right) \\
& \left(v^{q}(0)+p q \int_{0}^{t} b(s) \exp \left(-q \int_{0}^{s} c(\tau) d \tau\right) d s\right)^{\frac{1}{q}} \\
\leq & \exp \left(\int_{0}^{t} c(s) d s\right) \\
& \left(a^{p q}+p q \int_{0}^{t} b(s) \exp \left(-q \int_{0}^{s} c(\tau) d \tau\right) d s\right)^{\frac{1}{q}} \\
= & k(t) \exp \left(\int_{0}^{t} c(s) d s\right)^{\frac{1}{2}}
\end{aligned}
$$

Dimana $t \in J$ 
sehingga

$$
k(t)=\left(a^{p q}+p q \int_{0}^{t} b(s) \exp \left(-q \int_{0}^{s} c(\tau) d \tau\right) d s\right)^{\frac{1}{q}}
$$

Selanjutnya, substitusikan hasil di atas ke Persamaan (13) maka diperoleh

$$
u^{\prime}(t) \leq b(t) k(t) \exp \left(\int_{0}^{t} c(s) d s\right)
$$

Integralkan Persamaan (15) dari 0 ke $t$ terhadap kedua ruas sehingga diperoleh

$$
\begin{aligned}
& \int_{0}^{t} \frac{d}{d s}(u(s)) d s \leq \int_{0}^{t} b(s) k(s) \exp \left(\int_{0}^{s} c(\tau) d \tau\right) d s \\
& \left.u(s)\right|_{0} ^{t} \leq \int_{0}^{t} b(s) k(s) \exp \left(\int_{0}^{s} c(\tau) d \tau\right) d s \\
& u(t)-u(0) \leq \int_{0}^{t} b(s) k(s) \exp \left(\int_{0}^{s} c(\tau) d \tau\right) d s \\
& u(t)-\left(a+\int_{0}^{0} b(s)\left(u^{p}(s)+\int_{0}^{s} c(\tau) u(\tau) d \tau\right) d s\right) \\
& u(t)-(a+0) \leq \int_{0}^{t} b(s) k(s) \exp \left(\int_{0}^{s} c(\tau) d \tau\right) d s \\
& u(t)-a \leq \int_{0}^{t} b(s) k(s) \exp \left(\int_{0}^{s} c(\tau) d \tau\right) d s
\end{aligned}
$$

atau

$$
u(t) \leq a+\int_{0}^{t} b(s) k(s) \exp \left(\int_{0}^{s} c(\tau) d \tau\right) d s, \quad t \in J
$$

\section{KESIMPULAN}

Berdasarkan pembahasan, maka dapat diperoleh beberapa kesimpulan sebagai berikut :

1. Ketaksamaan integral Gronwall-Bellman merupakan salah satu teori ketaksamaan yang terdiri dari beberapa sifat, dimana sifat-sifat tersebut melibatkan beberapa fungsi yang didefinisikan sebagai fungsi tertentu dan nilai integral dari fungsi-fungsi tersebut. Dalam hal ini jika fungsi-fungsi tersebut terbatas terhadap nilai integral tertentu maka fungsi tersebut juga terbatas pada kondisi lain, yakni terhadap eksponensial dari integral tersebut.

2. Sifat dasar dari ketaksamaan integral GronwallBellman hanya melibatkan dua fungsi yang didefinisikan sebagai fungsi tertentu dan satu konstanta. Sifat dasar tersebut kemudian dapat dikembangkan dengan melibatkan lebih dari dua fungsi serta beberapa konstanta yang terbatas pada interval tertentu.

Di sisi lain, dengan menambahkan beberapa syarat lagi yaitu pangkat dari suatu fungsi atau konstanta, maka ketaksamaan integral tersebut menghasilkan beberapa sifat baru khususnya untuk fungsi berpangkat.

\section{DAFTAR PUSTAKA}

Bainov, Drumi. \& Simeonov, Pavel. (1991). Integral Inequualities And Applications, Kluwer Academic Publishers, USA.

Choy, Sung Kyu. Kang, Bowon. \& Koo, Namjip. (2007). On Inequalities Of Gronwall Type, hlm. 561 - 586, Department of Mathematics Chungnam University Daejeon 305-764, Republic of Korea.

Negoro ST. \& Harahap, B. (2005). Ensiklopedia Matematika, Ghalia, Bogor Selatan.

Oguntuase, James Adedayo. (2001). On An Inequality Of Gronwall, hlm. 1 - 6, Department Of Mathematical Sciences, University Of Agriculture, Abeokuta, Nigeria.

Owaidy, H. El , Ragab, A. \& Abdeldaim, A. (1999). On Some New Integral Inequalities of Gronwall Bellman Type, hlm. 289 - 303, Department of Mathematics, Faculty of Science, AL-Azhar University, Nasr - City.

Purcell, Edwin J. dkk. (2003). Kalkulus Jilid 1 Edisi Kedelapan, Erlangga, Jakarta.

Riyanto, M. Zaki. 2008. Pengantar Analisis Real I., Yogyakarta.

http://www.google.co.id/url?sa=t\&source=web\&cd=2\&ve $\mathrm{d}=0 \mathrm{CCEQFjAB} \& u r l=\mathrm{http} \% 3 \mathrm{~A} \% 2 \mathrm{~F} \% 2 \mathrm{Fwww} . e m i s$. de $\% 2$ Fjournals $\% 2 F D M \% 2 F v X I 2 \% 2 F a r t 4 . p d f \& r c t=j$ $\& q=$ integral\%20inequality \&ei=EFLZTc7ODI7evQP I-

PWkBw\&usg=AFQjCNGX8Hm1t4ZnSkpqNQGC MufH0qXUzw\&cad=rja.18 Mei 2011, Pkl. 21.45 WIT

http://en.wikipedia.org/wiki/Minkowski_inequality. Mei 2011, Pkl. 20.00 WIT 